\title{
Norois
}

Environnement, aménagement, société

$227 \mid 2013$

Sentir et ressentir la ville

\section{La notion de liberté comme concept analytique de la construction du rapport affectif des individus envers la ville}

The notion of freedom as analytical concept of individual's construction of affective relationship to the city

\section{Denis Martouzet}

\section{OpenEdition}

Journals

\author{
Édition électronique \\ URL : https://journals.openedition.org/norois/4685 \\ DOI : $10.4000 /$ norois. 4685 \\ ISBN : 978-2-7535-2916-8 \\ ISSN : $1760-8546$ \\ Éditeur \\ Presses universitaires de Rennes \\ Édition imprimée \\ Date de publication : 30 juin 2013 \\ Pagination : 69-80 \\ ISBN : 978-2-7535-2882-6 \\ ISSN : 0029-182X \\ Référence électronique \\ Denis Martouzet, «La notion de liberté comme concept analytique de la construction du rapport \\ affectif des individus envers la ville», Norois [En ligne], 227 | 2013, mis en ligne le 30 juin 2015, \\ consulté le 14 janvier 2022. URL : http://journals.openedition.org/norois/4685 ; DOI : https://doi.org/ \\ $10.4000 /$ norois. 4685
}




\title{
La notion de liberté comme concept analytique de la construction du rapport affectif des individus envers la ville
}

\author{
The Notion of Freedom as Analytical Concept of Individual's Construction \\ of Affective Relationship to the City
}

\author{
Denis Martouzet
}

IPAPE - UMR 6173 CITERES, Université de Tours, 33-35, allée Ferdinand-de-Lesseps - 37200 Tours,

(denis.martouzet@univ-tours.fr)

\begin{abstract}
Résumé : À partir des dires récoltés par différentes enquêtes menées sous forme d'entretiens visant à saisir le rapport à l'espace, notamment dans sa dimension affective, il est proposé une relecture transversale de ce matériau : le thème abordé est celui de la liberté, telle qu'elle est vécue et ressentie puis dite, renvoyant essentiellement au sentiment de liberté. La liberté est approchée sous la forme de l'autonomie et de la recherche d'autonomie puis sous celle de la revendication. Par la suite, sont analysés certains écrits de Jean-Jacques Rousseau pour montrer la complexité inhérente à cette notion et son importance dans la construction affective de l'individu, notamment dans le rapport affectif à la ville. Au-delà de la clarification des liens entre liberté et affectivité dans la construction du rapport à l'espace de l'individu, cette approche par la thématique de la liberté, parce qu'elle est transversale, permet de ne pas tomber dans le piège de la simplification des interactions entre les éléments constitutifs de ce rapport et la perte de sens qui y est associée.
\end{abstract}

\begin{abstract}
From statements collected through various inquiries in the form of conversations to understand the relationship to space, in particular in its emotional dimension, we propose a new transverse analysis of this material: the issue dealt with, is the one of freedom, such as it is experienced, felt expressed, and relates back essentially to the feeling of freedom. This freedom is approached in terms of autonomy and the search for autonomy, then in the form of assertion. Subsequently, we analyze certain texts by Jean-Jacques Rousseau to show the complexity inherent to this notion and its importance in the emotional construction of the individual, in particular in the emotional relationship to the city. Beyond the clarification of the links between freedom and affectivity in the construction of the individual's relationship to space, this approach through the theme of freedom, because it is transverse, allows us not to fall into the trap of simplifying the interactions between the constituent elements of this relationship and the loss of meaning associated with this.
\end{abstract}

Mots clés : liberté - ville - rapport affectif à la ville - Jean-Jacques Rousseau

Keywords: freedom - city - affective relationship to the city - Jean-Jacques Rousseau

Les éléments de connaissance développés ici participent de la question des modalités de construction du rapport affectif de l'individu envers la ville. Nous avons présenté par ailleurs (Martouzet, 2007, 2008,
2010), dans le cadre de cette problématique, des résultats obtenus à partir de l'analyse d'entretiens ouverts effectués auprès de plusieurs échantillons de personnes habitant, au sens fort du terme, des 
espaces urbains et/ou périurbains ${ }^{1}$. Par des jeux de déconstruction et de reconstruction des discours recueillis, il a été possible, entre autres, de faire émerger des figures de rapport à la ville ou des figures de ville en tant qu'elles sont affectivement appréciées ou dépréciées. D'autres analyses ont permis de clarifier les grandes catégories de facteurs entrant dans cette construction, entre aménités et urbanité (Bochet, 2000), entre civilité et lisibilité (Lynch, 1960; Martouzet, 2007, 2008). D'autres encore ont mis en évidence les interactions entre ce qui relève des sens et de la sensibilité, du plaisir et du déplaisir qui y sont associés, ce qui relève des représentations et des pratiques de l'espace urbain, par le moyen du concept de mode d'habiter (Mathieu, 1996, 2006, 2007 ; Morel-Brochet, 2006, 2007; Morel-Brochet et Ortar, 2012), amenant à définir le rapport affectif à la ville comme l'ensemble fluctuant des relations qu'entretient un individu envers la ville, à travers les représentations qu'il s'en fait pour peu que ces relations mettent en jeu, ponctuellement (émotion) ou durablement (sentiments) le soi, conduisant à des représentations valorisantes ou dévalorisantes de la personne pour et par elle-même. D’autres, enfin, ont permis de préciser les moments forts constitutifs de cette construction, parmi lesquels les premières fois (Martouzet, 2010). De ce fait, cet ensemble de recherches et donc le présent texte se positionnent comme étant, parallèlement, la recherche d'un équilibre individuespace ou habitant-milieu, refusant de choisir entre sciences de l'espace et sciences de l'individu ou des groupes, quelle que soit l'échelle (sociologique, sociopsychologique, psychologique). Plus encore, le positionnement choisi récuse la personnification de la ville et le réalisme spéculatif appliqué, à la Thrift (Amin et Thrift, 2002). L'équilibre recherché

1. Nous ne développons pas ici, de nouveau, les aspects méthodologiques et techniques liés aux modalités de constitution des échantillons et à la mise en œuvre de ces enquêtes. Dans les grandes lignes, il s'agit d'entretiens ouverts, longs portant, pour certains, sur le rapport à l'espace et l'habiter, pour d'autres, sur le rapport affectif à l'espace. Certains de ces entretiens ont été réactivés par une méthode particulière originale (Martouzet et al., 2010) visant, autant que possible, les aspects intimes de ce rapport à l'espace, par une technique permettant l'association d'idées plutôt que la mise en récit. Ces entretiens ont été réalisés auprès d'adultes, à l'échelle temporelle de leur vie, ce qui a amené la plupart d'entre eux à évoquer, décrire ou préciser leur enfance ou certains moments de celle-ci.Pour plus de détails sur les entretiens menés, se reporter au rapport de recherche Ehea - Espaces habités, espaces anticipés : http://citeres.univ-tours.fr/ spip.php?article1238. Pour plus de détails sur le plan méthodologique et sur le plan épistémologique de la relation entre discours et pratiques, en plus du rapport pré-cité, le lecteur peut aussi se reporter aux travaux de thèse de Benoît Feildel (2010) et de Nathalie Audas (2011). se situe plutôt dans la perspective relationnelle de Laflamme $(1995)^{2}$.

À présent, et suite à ces déconstructions-reconstructions, nous évoquons comment un thème transversal, celui de liberté, permet une nouvelle interprétation des discours recueillis et plus largement contribue à la compréhension du rapport affectif à la ville (Martouzet, 2007, 2008, 2010), aux lieux urbains (Audas, 2011) et, plus généralement, à l'espace (Feildel, 2010), ce qui ne remet pas en cause les résultats précédemment obtenus mais qui, au contraire, les met en perspective les uns par rapport aux autres et permet d'éclairer ainsi les mécanismes en œuvre dans la construction et l'évolution du rapport affectif à la ville. Quelques précisions sont utiles, relatives à l'utilisation qui est faite de la notion de liberté. Tout d'abord, la liberté, dont nous faisons l'hypothèse qu'elle entre dans la construction du rapport affectif à la ville, n'a pas été pensée comme constitutive, au départ, de l'objet de recherche, des questions de recherche construites pour la compréhension de cet objet, ni prise en compte, a priori, sur le plan méthodologique et, notamment, sémantique. Cette notion apparaît plutôt comme entrée constitutive d'une grille qui reste à élaborer pour l'analyse des discours recueillis et héritière même de ces discours. Il nous est apparu, en effet, à de nombreuses reprises si ce n'est pas de façon systématique, que la liberté, bien que le terme n'ait été que très rarement prononcé par les personnes interviewées, sous-tend une grande partie de cette construction affective de l'individu, dont nous allons développer ici, principalement, la dimension spatiale, dans le contexte particulier des espaces urbains. D'autre part, il ne s'agit pas ici de chercher à définir la liberté, ce qui n'est pas de notre ressort ni de nos compétences, ni sur le plan philosophique, ni sur le plan juridique. De plus, alors même que les pratiques et les représentations urbaines renvoient à des conceptions particulières de la liberté et de son expérience, nous n'abordons pas ici l'analyse interculturelle de ces pratiques et de ces représentations qui pourrait, comme d'ailleurs

\footnotetext{
2. Plutôt qu'un état de l'art de la thématique spatiale à travers les affects que les lieux, de tous types et de toutes échelles, peuvent générer (rapport affectif à la ville) ou abriter (rapport affectif dans la ville), renvoyant à la littérature géographique anglo-saxonne avec en premier lieu Tuan ou Thrift, ainsi qu’à la philosophie phénoménologique et ses déclinaisons géographiques (Paquot et al., 2007) par exemple ou la psychologie environnementale, notamment par la notion d'attachement, nous renvoyons notamment à Feildel (2010), Audas (2011) et Martouzet (2014).
} 
leur analyse historique, s'avérer nécessaire en vue d'une certaine exhaustivité. Au contraire, nous envisageons la notion de liberté comme très extensive et si la liberté peut apparaître, pour certains, comme une quête philosophique, elle n'est pour d'autres qu'un ressenti plus ou moins exprimable, sous l'expression assez floue de "sentiment de liberté $»^{3}$. C'est l'expression de ce sentiment qui nous a été en partie accessible dans les entretiens menés et l'exemple que nous développons plus loin de JeanJacques Rousseau permet une double mise en perspective, du fait de son ancrage dans une société différente de la nôtre mais qui en pose les bases philosophiques et les prémisses sociologiques et par le fait que Rousseau n'est pas un promeneur ordinaire (Martouzet, 2014) : ses pratiques de la ville ne sont pas celle de tous.

Nous abordons la liberté dans un premier temps par les notions d'autonomie et d'autonomisation de la personne puis comme revendication. Le troisième point porte sur la complexité du rapport affectif à la ville, c'est-à-dire de sa capacité à être paradoxal (dans le sens d'auto-contradictoire) faisant l'hypothèse que ce sont, entre autres, les différentes dimensions de la liberté qui, conjointement, amènent cette complexité.

\section{LA RECHERCHE D'AUTONOMIE ET LA CONSTRUCTION DU RAPPORT AFFECTIF ENVERS LA VILLE}

Dans un premier temps, nous considérons la liberté comme une autonomie acquise, acquise par rapport à autrui duquel, à certains moments de la vie, l'individu peut être dépendant et acquise par soi, c'est-à-dire par le développement des capacités de la personne, à mesure que celle-ci avance en âge. La dimension temporelle est donc ici première, tout comme elle a été centrale dans la recherche de la compréhension des processus en œuvre, à partir des émotions, des pratiques, des habitudes acquises ou en cours d'acquisition, des apprentissages, etc. dans la construction jamais achevée du rapport affectif de

\footnotetext{
3. Nous utilisons par la suite le seul terme de liberté, alors même qu'il s'agit généralement de ce sentiment de liberté ou, du moins, la liberté en tant que le ressenti qui en est fait passe par les mots dits lors des entretiens. C'est, plus précisément, tout à la fois une liberté ressentie et une liberté vécue qui peuvent l'une comme l'autre se situer à l'intersection de l'expérience personnelle et de la conception philosophique, plus ou moins claire et mobilisée de façon plus ou moins consciente, de la personne elle-même.
}

l'individu envers la ville. Il est aussi apparu que cette construction, loin d'être linéaire et régulière, s'appuie, d'une part, sur des habitudes et des apprentissages mais, principalement, sur des moments forts et, parmi ceux-ci, les «premières fois » sont primordiales. Primordiaux car déterminants et déterminants car initiant un processus, ce sont des moments intenses de cristallisation de ce rapport : le souvenir en reste vivace longtemps après et l'habiter des individus adultes en découle en grande partie.

La notion de «première fois » reste souple : il faut y voir l'idée de nouveauté expérimentée, en tant que cette nouveauté provoque émotion et/ou sentiment conduisant à la mise en évidence, y compris pour celui qui vit cette "première fois ", d'un décalage irréductible entre un «avant » et un « après », décalage dont les conséquences, de façon consciente ou non, sont encore d'actualité, puisque évoquées dans les discours.

Une situation de "première fois », telle qu'ainsi précisée, peut être vécue lors de la première rencontre avec une ville particulière, pouvant provoquer une émotion puis susciter un sentiment durable, structurant alors les affects de la personne et, notamment, son rapport affectif envers cette ville en particulier mais aussi son rapport affectif à la ville (à la ville en général). Plus structurant s'avère être non la rencontre avec une ville mais avec la ville, avec l'espace urbain et sa diversité. Il s'agit essentiellement des premiers allers et retours entre le domicile et l'école, lorsque ceux-ci ne sont pas effectués - enfin! diraient de nombreux enfants - sous l'autorité parentale, aussi bienveillante soit-elle, par les parents eux-mêmes ou, par procuration, par une autre personne (grand frère, grande sœur, voisin, parent d'un autre élève...). Ce sont aussi, à l'adolescence ou à l'approche de celle-ci, les premières sorties avec les copains puis ce sera, quelques temps plus tard, les années d'études dans une ville plus ou moins éloignée du lieu de résidence familiale et qui amène, souvent oblige à avoir son premier chez-soi, au prix de négociations quant à la localisation dans la ville, la surface, le montant du loyer...

Dans ces situations, et sans doute dans d'autres, on repère un point commun : c'est un moment important d'acquisition d'un certain degré d'autonomie vis-à-vis de l'autorité de référence. Y est associé l'effet de nouveauté qui est la marque d'un aboutissement, celui d'avoir atteint, après avoir gravi 
différents échelons et passé par plusieurs phases, un premier degré ou un degré supplémentaire.

On constate aussi que le contenu même de ce moment - ce qui est vu et expérimenté de la ville, des autres et de soi - n'est que très peu important : ce qui compte est l'absence du regard habituel de l'autre, des parents notamment. C'est cette absence même, créant un vide, qui permet à l'enfant de remplir ce moment, entièrement, sans contrainte - du moins le croit-il, ce qui suffit pour qu'émerge le sentiment de liberté - par le soi qui peut ainsi se développer, se répandre. La ville, elle, parce qu'elle participe du contenu de ce moment, est la structure qui permet le développement de ce moment, par les multiples possibilités qu'elle offre et qui apparaissent nouvelles, les actes ainsi possibles concrétisant le sentiment de liberté et ancrant la liberté elle-même dans la réalité. Peu importe le flacon, il y a l'ivresse. Vu avec des yeux d'adultes, par quelqu'un d'extérieur ou par l'enfant devenu grand, c'est-à-dire avec une certaine objectivité, du moins une certaine relativité, ces moments intensément vécus, ce chemin de l'école, ces sorties, etc. sont d'une grande banalité : ce sont les mêmes pour des milliers d'enfants, quel que soit, d'ailleurs, leur ancrage (urbain, rural) et d'une façon générale les circonstances spatiales. La personne elle-même les vivra plusieurs fois à mesure de l'avancée en âge, mais si le contenu est identique ou semblable, la qualité affective (tonalité affective, négative ou positive, et intensité) ne sera plus la même. L'expérience de la "première fois ", en fait des «premières fois » puisque la première " première fois » n'épuise pas le potentiel de nouveauté ni de ressenti, est marquante, quelles qu'en soient les circonstances.

Dans les exemples donnés, qui sont les plus souvent cités dans le système de justifications qu'apporte l'individu à son rapport affectif à la ville, s'esquisse une gradation qui, elle aussi, n'a que peu à voir avec le contenu urbain relatif aux différentes expériences. Cette gradation se décline en fonction de l'âge mais aussi de l'éloignement spatial et de la durée d'éloignement. Si un certain niveau d'autonomie peut être définitivement acquis, le sentiment de liberté relatif à cette autonomisation semble, pour perdurer, nécessiter une accentuation par à-coups plutôt que progressive : le potentiel de nouveauté et de ressenti s'amenuise, voire disparaît avant un nouveau type d'expérience en ville.
C'est bien le caractère nouveau des situations qui importe, mais toute nouveauté n'imprime pas aussi fortement sa marque sur la construction affective de l'individu. Ici, la nouveauté porte sur l'expérimentation de la liberté en tant qu'elle est ressentie, c'està-dire l'expérience du sentiment de liberté, parfois même l'anticipation de celui-ci comme en atteste les demandes récurrentes de l'enfant pour aller seul à l'école. Quels qu'en soient les éléments conceptuels mobilisés, quels qu'en soient les souvenirs, éventuellement les attentes, du moment qu'ils se traduisent par le sentiment d'une liberté reconnue comme telle, trois dimensions interviennent dans la compréhension de l'importance de la liberté dans la construction du rapport affectif à la ville : le rapport à l'autre, le rapport à soi et le rapport à la ville.

Le rapport à l'autre est d'abord à analyser en termes d'éloignement relativement aux représentants de l'autorité que sont les parents. Il ne s'agit pas tant d'être en ville que de ne pas être sous le regard parental. Pouvoir ne plus être sous ce regard indique à l'enfant ou à l'adolescent la relativité du besoin d'être avec ses parents, d'avoir des parents. C'est une première forme d'émancipation qui, audelà des aspects matériels, est une façon de dire aux parents et au monde le fait d'être grand et revendiquer d'être reconnu comme tel.

La situation est néanmoins paradoxale. D’une part, se sentir obligé de "faire le grand », de l'affirmer, de vouloir être reconnu comme tel est la preuve même de ne pas l'être tout à fait : le grand n'a pas besoin de jouer au grand. Une part de mauvaise foi, au sens de Sartre (1999), est présente ${ }^{4}$ et celle-ci peut expliquer le surgissement de l'émotion et l'imtrès particulière de mensonge : elle a « en apparence la structure du mensonge. Seulement ce qui change tout, c'est que dans la mauvaise foi, c'est à moi-même que je masque la vérité » (1999, p. 84). Il ne s'agit donc pas de conférer à cette expression le sens habituel du langage courant (la malhonnêteté de quelqu'un qui affirme des choses qu'il sait fausses ou qui feint l'ignorance qui s'oppose en partie à la bonne foi considérée comme l'attitude de quelqu'un qui parle ou qui agit avec la conviction d'être honnête, de respecter la vérité, ce qui n'exclut pas l'erreur, mais seulement l'erreur faite exprès, comme l'indique la définition juridique de la bonne foi : « croyance erronée en l'existence d'un droit ou d'une règle juridique, par ignorance ou suite à une tromperie " (Larousse). La définition de Sartre ne correspond donc pas à celle du langage commun et même s'y oppose puisque celui qui fait preuve de cette mauvaise foi sartrienne le fait en toute bonne foi. « Le véritable problème de la mauvaise foi vient évidemment de ce que la mauvaise foi est foi » (1999, p. 104). En effet, « la dualité du trompeur et du trompé n'existe pas ici » (1999, p. 84). « Il s'ensuit d'abord que celui à qui l'on ment et celui qui ment sont une seule et même personne, ce qui signifie que je dois savoir en tant que trompeur la vérité qui m’est masquée en tant que je suis trompé. Mieux encore, je dois savoir très précisément cette vérité pour me la cacher plus soigneusement - et ceci non pas à deux moments différents de la temporalité - ce 
pact de cette expérience dans la construction du rapport affectif à la ville. D'autre part, la situation de première fois urbaine, dans laquelle il s'agit de s'affirmer et de montrer que l'on s'affirme, convoque deux publics : les personnes de connaissance (voisins, instituteurs ou professeurs, copains...) dont la probabilité de rencontre, bien que faible, n'est pas nulle, et des inconnus beaucoup plus nombreux. Ce sont ces derniers, par leur caractère d'inconnus, sans une quelconque autorité, si ce n'est celle de l'adulte aux yeux de l'enfant, et par leur nombre qui permettent d'expérimenter l'anonymat urbain, composante typique du mode de vie urbain, d'être un anonyme parmi les anonymes. Pourtant, la valorisation de soi par le fait d'être reconnu comme appartenant au monde des adultes passe d'abord par le regard des personnes de connaissance, ceux-là même qui mettent à mal l'anonymat. On a donc ainsi deux phénomènes qui s'entrecroisent et se renforcent : l'expérimentation de l'anonymat et la reconnaissance, par les non anonymes, du pouvoir d'être anonyme, c'est-à-dire l'affirmation de soi, la revendication d'être inclus dans la société. Ce rapport à l'autre est donc bien d'abord le miroir du regard de l'autre que l'on porte sur soi : ce n'est pas le regard de l'adulte anonyme qu'on croise qui compte véritablement mais la représentation que s'en fait l'enfant ou l'adolescent. Enfin, il y a la découverte d'autres « autres », la découverte que la diversité humaine et sociale dépasse largement le cadre de ce qui est déjà connu : le cercle familial, l'univers scolaire.

On voit, dans ce qui précède, que la ville, dans sa dimension concrète, matérielle n'est pas un élément spécifique de la construction de soi : ce type d'expérimentation peut tout aussi bien se faire en d'autres types de lieux. Elle n'est, là, que décor, mais on a montré ailleurs (Martouzet, 2008) combien la ville en tant que décor, pour peu que s'y déroule un événement non spécifique à la ville fortement chargé affectivement, se voit attribuée la charge affective de cet événement qui aurait pu advenir n'importe où ailleurs.

Le rapport à soi passe essentiellement par la valorisation de soi permise par les expériences nouvelles y compris l'expérience du sentiment de liberté décrit précédemment. Il ne s'agit, par exemple, pas tant d'être grand ou faire grand aux yeux du monde

qui permettrait à la rigueur de rétablir un semblant de dualité - mais dans la structure unitaire d'un même projet » (Sartre, 1999, p. 84-85). (d'ailleurs le monde ne s'y trompe pas), qu'à ses propres yeux, ce qui, là, conduit à la valorisation affective du moment de liberté et, par ricochet, du lieu de ce moment. Existent aussi des moments de dévalorisation quand la ville ne permet pas, quand l'enfant n'ose pas... C'est aussi la valorisation de soi par la mise en œuvre de capacités acquises et en cours d'acquisition par l'expérience, associée au plaisir de cette mise en œuvre : sur le plan spatial, c'est la capacité de se repérer dans la ville ou un morceau de celle-ci, d'aborder un inconnu et par là même de se confronter à l'inconnu ou de s'affranchir, à l'âge de la transgression des règles qu'est l'adolescence, du respect de certaines d'entre elles, de façon plus ou moins provocatrice.

Cependant, si la ville n'est pas le seul lieu d'expérimentation de soi, par sa diversité, elle est un lieu idéal pour cette autonomisation. En effet, elle offre un certain nombre de possibilités matérielles mais aussi de rencontres, de surprises, qui, toutes, sont autant d'opportunités de mise en œuvre de ces nouvelles capacités et autant de prétextes pour s'éloigner du regard parental, sans toutefois que le risque, l'insécurité ou le sentiment d'insécurité soient trop importants, notamment du fait du caractère normé de la ville, de la relative faiblesse de l'éloignement: si besoin est, rentrer dans le giron familial est chose facile du fait du faible éloignement, qui néanmoins augmente avec l'âge, de facilités de transport, de l'utilisation du téléphone portable. C'est l'aventure bien cadrée.

Plus tard la ville permettra l'émancipation, puis l'autonomie, voire la totale indépendance, notamment sur le plan financier : c'est elle qui fournira les moyens de subsistance à travers le ou les premiers emplois qu'elle procurera. Ainsi, la ville construira l'individu et fera entrer, plus ou moins parfaitement, celui-ci sous l'une ou l'autre des figures que nous avons pu mettre en évidence : celle du convaincu, celle du libéré qui réfèrent directement à cette question d'autonomisation, et plus indirectement l'amoureux ou celui qui sait se saisir des opportunités urbaines ${ }^{5}$ (Martouzet, 2007).

\footnotetext{
5. Parmi l'ensemble de figures élaborées par ailleurs, celles qui sont présentées ici renvoient aux personnes pour qui la ville a, plus ou moins, construit l'individu : le « convaincu » est celui qui, bien qu'au départ n'appréciant pas ou peu la ville, se laisse peu à peu convaincre et finit par lui trouver du positif, y compris sur le plan des affects ; le « libéré » est celui qui a, justement, pu acquérir son autonomie par la ville (par l'éloignement - de l'autorité parentale mais aussi des contraintes spatiales de son environnement antérieur -, par les possibilités matérielles ou non qu'elle
} 
Si l'air de la ville rend libre, si la ville est un «terrain de jeux » permettant ou favorisant peu à peu l'autonomie de la personne, ce qui lui confère un sentiment de liberté et participe, plus largement, à la construction du rapport affectif à la ville, on pourrait, parce que d'autres configurations spatiales, moins diverses peut-être, le permettent aussi néanmoins, faire la même analyse avec d'autres types d'organisation spatiale. On peut illustrer cela par le roman de Louis Pergaud, La guerre des boutons, et ses traductions cinématographiques, notamment celle d'Yves Robert, qui montrent bien une alternance de scènes de liberté la plus débridée et de scènes où, parce que les deux mondes, celui des adultes et celui des enfants, celui du village et celui des champs et des bois, ne sont pas suffisamment étanches aux yeux des enfants, l'autorité parentale s'exprime de façon constante et parfois brutale.

\section{LA LIBERTÉ COMME REVENDICATION OU « IL VAUT MIEUX SE SENTIR LIBRE ET SE DIRE LIBRE »}

$\mathrm{Si}$, dans ce qui précède, la liberté fait l'objet d'une recherche, plus ou moins active, plus ou moins volontaire, ou d'une expérience dans laquelle l'individu découvre malgré lui, voire " par surprise ", si ce n'est la liberté elle-même du moins un sentiment de liberté qui met en jeu le soi (découverte de ses propres capacités, découverte de l'intérêt de les exercer...), les autres (autonomisations par rapport aux tutelles) et la ville (à la fois comme moyen, comme lieu et comme décor de cette autonomisation et du sentiment de liberté qui l'accompagne), la liberté peut être aussi l'objet d'une revendication. Cela signifie que nous entrons dans le domaine des discours et que nous quittons celui des faits, bien que ceux-ci aient été analysés via des dires. Il y a maintenant, au-delà de l'information, intention dans le discours, ce qui suppose une posture de la part de l'individu qui envisage la liberté non pas comme le résultat plus ou moins fortuit d'expériences mais comme valeur. La revendication, même implicite, même inconsciente est un positionnement de l'indi-

offre) ; l'« amoureux » se laisse construire affectivement par la ville qu'il se construit affectivement; l'« opportuniste » se construit affectivement par ce qu'il saisit de la ville et, donc, par les prises qu'elle offre (Gibson, 1986; Audas, 2011). D'autres figures renvoient soit à l'idée d'une réticence à la ville (le « rétif »), soit à une absence d'affectivité (l'« utilitariste ») ou encore à une ville qui n'existe plus (le «nostalgique»). vidu dans et face à la société. De plus, elle exprime un manque : le seul sentiment de liberté préalablement décrit et, pour l'individu, précédemment acquis, n'est pas ou n'est plus suffisant, il faut aussi l'affirmer.

Cependant, un problème d'ordre logique et méthodologique apparaît. La revendication de la liberté n'est acceptable, aux yeux de celui qui revendique, que si cette liberté est une valeur collective dans le sens où, non seulement c'est une valeur partagée mais aussi dans le sens où la revendication ne se fait pas seulement au nom de l'individu mais pour un collectif. Revendiquer la liberté pour d'autres que soit est possible (l'individu aide, il se situe « au-dessus » des autres), revendiquer pour tous est, aussi, possible (l'individu se situe « au même niveau » que les autres), revendiquer pour soi est plus difficile : cela revient, dans le cas qui nous intéresse ici, à admettre que l'on n'est pas libre, ce qui, si cela peut refléter une certaine clairvoyance, est d'abord une dévalorisation de soi aux yeux de la société et, surtout, une autodévalorisation, c'est-à-dire à ses propres yeux. Aussi, lors d'entretiens, pendant lesquels peut survenir une tendance à se mettre en valeur, l'expression de la liberté, comme valeur personnelle partagée, en tant qu'elle est revendiquée, ne peut que difficilement être recueillie, elle ne peut être directement atteinte : ne pas aborder le sujet est le meilleur moyen de l'atteindre, à l'image des effets essentiellement secondaires de Elster $(1986)^{6}$.

Dans les recherches précédemment évoquées, visant à connaître la dimension affective du rapport qu'entretient l'individu envers la ville ou envers les lieux et sans que soit abordée, à aucun moment, la question de la liberté, il est apparu à au moins deux reprises que les discours donnés à entendre avaient en toile de fond cette idée de liberté non comme conséquence d'expériences survenant avec l'avancée en âge, mais comme discours à tenir, quel que soit le degré de vraisemblance avec la réalité vécue. Les deux exemples que nous présentons marquent, selon nous, la nécessité d'un recours à des post-rationalisations fondées sur une réécriture de l'histoire personnelle permettant de se valoriser ou, au moins, de ne pas devoir se dévaloriser aux

\footnotetext{
6. De façon succincte, les effets essentiellement secondaires correspondent aux objectifs que l'on ne peut pas se fixer, car le seul fait de vouloir les atteindre empêche d'y parvenir : un individu ne peut se forcer à dormir, ni à oublier, ni à être spontané, par exemple.
} 
yeux de l'autre. Le décryptage de ces post-rationalisations montre que la liberté individuelle est un discours que la société nous enjoint de tenir, quitte à tordre la réalité, et que derrière le discours se tient une revendication envers la liberté, même si celle-ci relève de l'illusion plus que des faits.

Dans le premier cas, l'hypothèse avait été faite d'une corrélation positive entre la durée cumulée de fréquentation de la ville (logement, loisir, travail, etc.), rapporté à l'âge de la personne, et l'évaluation affective de la ville, comme si l'apprentissage de la ville était un facteur déterminant dans la construction du rapport affectif à la ville, sa valeur et sa valence. Retranscrite sous forme graphique, cette hypothèse a été présentée aux enquêtés comme un résultat, encore provisoire mais déjà assez fermement établi, de manière à pouvoir solliciter l'avis de l'enquêté : il lui était ainsi demandé ce qu'il pensait de ce nuage de points structuré par une droite de corrélation positive. La plupart des personnes enquêtées, répondant à cette demande de formulation de leur avis sur ce graphique, n'ont pas proposé une analyse mais, directement, une interprétation, parmi les deux possibles : premièrement, "plus on est en ville, plus on aime la ville », celle-ci étant l'expression de l'hypothèse de l'apprentissage (hypothèse qui s'est révélée sans aucune valeur) et, deuxièmement, «plus on aime la ville, plus on est en ville (étant sous-entendu que plus on aime la ville, plus on y va, plus on y reste). Sans exception, toutes les personnes interrogées ont directement, clairement et exclusivement, chacune à leur manière quant à la formulation, interprété le graphique selon la deuxième proposition.

L'analyse que nous faisons de cette série d'interprétations allant toutes dans le même sens est la suivante : la première proposition «plus on est en ville, plus on aime la ville » est comprise comme l'idée qui voudrait que l'individu dans ce qu'il a de plus personnel comme mode de justification (j'aime) ne serait finalement que le résultat de ce que son contexte, en l'occurrence la ville, contexte qui lui échappe nécessairement en grande partie, a « déversé » en lui. Ainsi l'individu n'est que le résultat de facteurs qui lui sont extérieurs : la détermination est forte, l'individu est produit, il n'est pas libre. A contrario, l'autre proposition révèle un choix qui est de l'ordre de l'évidence : «Puisque j'aime la ville, c'est normal que j'y sois (souvent). » Le soi détermine les pratiques : la détermination est aussi forte mais elle est, à l'échelle de l'individu, autodétermination, donc autonomie. Dire cela revient à se dire comme libre. Mais implicitement et paradoxalement $^{7}$, le fait de revendiquer, et non simplement de dire, révèle qu'on ne l'est pas.

À partir de cet exemple, contrairement à celui qui suit, il n'est pas possible de savoir si cette liberté est revendiquée parce qu'elle est ressentie comme constitutive de la personne par elle-même, ou parce que son expression traduit un manque qu'il s'agit de masquer aux yeux d'une société qui valorise la liberté individuelle et, par conséquent, l'individu libre.

Le second exemple porte sur un échantillon de personnes logeant dans le périurbain. Là encore, la question de la liberté n'avait pas été abordée, ce n'était pas un objectif de la recherche. Il était question de rechercher en quoi leur spatialité à l'échelle biographique contenait des éléments de compréhension du fait de loger là où ils logeaient. Ces périurbains, lors des entretiens ouverts et détaillés auxquels ils ont bien voulu se soumettre, ont, pour la plupart, dans un premier temps, eu un discours d'évaluation de leur localisation qui, en dehors des détails liés aux contextes précis de chacun, peut se résumer par l'idée que le périurbain est la localisation idéale puisqu'il conjugue les avantages de la ville et ceux de la campagne sans imposer les inconvénients de la ville, ni ceux de la campagne, au point que, pour certains, habiter ailleurs semblait relever de l'aberration. Il s'agit bien entendu, au-delà du discours très rationalisant, d'une évaluation subjective, les avantages et inconvénients respectifs n'ayant pas lieu d'être ici discutés, et affective ainsi que l'a clairement montré la tonalité des discours recueillis, impliquant un enjeu de soi, c'est-à-dire mettant en jeu l'identité de soi. Si l'on reprend les catégories d'analyse appliquées au précédent exemple, il est aisé de dire « ici, c'est bien, mieux qu'ailleurs, je suis "quelqu'un de bien”, par conséquent j'ai choisi de loger en cet endroit ». En revanche, il est beaucoup plus difficile d'avouer et d'assumer le fait que loger en tel endroit est la conséquence de causes financières et de contraintes liés aux lieux et aux horaires de travail des adultes du ménage.

\footnotetext{
7. Le paradoxe réside dans le fait que revendiquer sa liberté n'est pas possible, comme indiqué précédemment, aussi ne peut-on que la dire, mais la dire (quand, au final, on sait qu'elle n'est pas effective ou du moins pas sure) revient à la revendiquer.
} 
Or, la suite des entretiens, faisant appel à l'ensemble de la spatialité de l'individu, a amené à remobiliser des représentations relatives à d'autres lieux, d'autres types de lieux que le périurbain. Au final, tout bien considéré, la très grande majorité des personnes préfèrerait loger en ville, quelques autres à la campagne, mais pas dans le périurbain. Il ne faut pas voir là une critique du périurbain, ni du fait d'y loger, encore moins une critique des dires des personnes interrogées, mais y voir le fait que cette revendication n'est pas toujours une affirmation positive de soi, plutôt l'expression d'un manque que non seulement il faut cacher mais que, de plus, il faut convertir positivement : c'est un mensonge actif, non par omission, un mensonge aux yeux de l'enquêteur et aux yeux de la société que ce dernier représente et, surtout, un mensonge à soi-même (Dupuy, 1996). L'individu adhère donc à la valeur, socialement partagée, de la liberté individuelle au point de l'intégrer si ce n'est dans les actes du moins dans les discours de justification des actes lorsqu'il est dans l'obligation d'en donner un sens, alors même que l'explication que peut fournir l'analyse de l'ensemble du discours ne correspond pas à cette justification.

Notre interprétation est donc concordante avec celle exprimée à propos du premier exemple, celui de l'hypothèse d'une corrélation positive entre la durée cumulée de fréquentation de la ville et l'évaluation affective de la ville : il y a bien post-rationalisation. La différence réside dans le fait que, dans ce second exemple, la revendication est sous-tendue par un manque (que révèle la suite du discours), ce qui n'était pas sûr dans le premier cas.

Les discours d'affirmation de soi est sans doute plus aisé à prononcer aujourd'hui, plus acceptable que quelques décennies plus tôt. Sans entrer dans le vaste champ des considérations sociétales relatives à l'évolution récente du rapport de l'individu à la société depuis un demi-siècle, nous pouvons simplement signaler que les discours sur l'aliénation des masses et des individus ne sont plus à l'ordre du jour, du moins n'apparaissent-ils plus au premier plan. En parallèle, la société occidentale actuelle, jusqu'à ses avatars et ses zones d'influence les plus éloignées, se caractérise, entre autres, par le phénomène d'individuation (Martuccelli, 2005), qu'il faut bien envisager à deux niveaux qui se renforcent mutuellement. Premièrement, on constate une volonté accrue, de la part des individus, d'une plus grande affirmation de soi qui se traduit dans les actes, les prises de position, les discours, les attitudes et, préalablement, les choix conscients ou inconscients qui y ont présidé. Deuxièmement, il y a de la part de la société, envers l'individu, une véritable injonction à l'individuation qui se traduit tout autant dans l'offre en matière de modes vestimentaires, d'achat immobilier, de destination touristique, de la consommation en général, mais aussi dans les possibilités de communiquer : «Dans le futur, chacun aura droit à 15 minutes de célébrité mondiale » avait annoncé Andy Warhol. Il ne s'agit pas tant de pouvoir se différencier de l'autre quant aux résultats des choix effectués, ce qui est limité quand l'offre est, elle-même, limitée voire très limitée dans sa diversité par rapport au nombre d'individus, que de montrer que les choix faits sont l'expression de l'affirmation de soi et renvoie à l'idée d'autoréférencement. Or, s'il existe, schématiquement, deux grands modes délibératifs individuels, que sont la raison et les affects, l'une, la première donne moins à voir la possibilité et la capacité d'autodétermination. En effet, choisir telle option rationnellement signifie que toute autre personne ayant les mêmes besoins ferait le même choix et que ce même choix découle, au-delà des besoins, des circonstances, par nature extérieures à la personne. En revanche, les affects ou plus simplement le fait de dire «j'aime » ou « je fais cela parce que j'aime... » non seulement ne réfère pas à l'extériorité, mais, de plus, suffit comme argumentation.

La rationalité ne permet qu'une manière d'être (dans un contexte donné le résultat sera toujours le même) alors que la non-rationalité, dans laquelle se positionne la "délibération affective ${ }^{8}$ ", ouvre tous les possibles. Le choix étant ouvert, la délibération devient expression d'une liberté autoréférente. L'injonction à l'individuation conduit donc non à faire tel choix parmi les options possibles mais à justifier le choix fait par ce qui ne relève pas du rationnel $^{9}$. Ce qui compte est la valorisation de soi, aux yeux d'autrui et à ses propres yeux, à travers l'image d'être délibérant de soi que l'on veut donner. Un choix effectué aux yeux du monde est plus valorisant si, dans cette société d'individuation, il ne nécessite

8. L'expression peut sembler paradoxale. Elle signifie la capacité des individus de faire avec leurs émotions, de les manipuler, de se laisser manipuler par elle.

9. A moins d'être affectivement porté sur la rationalité et de l'affirmer comme un mode délibératif rationnel choisi non rationnellement. 
pas un «parce que » rationnel, ni une explication ayant forme logique mais s'affirme par un «j'aime et c'est ainsi » qui ne suppose pas de discussion à suivre : «c'est mon choix ${ }^{10}$ ! » devient l'affirmation même de la liberté telle que pensée par beaucoup.

Enfin, la rationalité, reposant sur la logique et la compréhension des relations de cause à effet, suppose la possibilité d'une erreur (de logique, d'appréciation de la réalité) par le fait même qu'elle se situe dans la raison. Le «j'aime » lui, ne peut contenir d'erreur, l'erreur n'étant pas de son ordre, même si le résultat du choix reste éventuellement discutable.

Plusieurs précisions s'imposent. Il va de soi que rationalité et affectivité ne sont pas aussi radicalement distinguables. Les liens multiples ont été montrés tant en philosophie (Hume, 1991; Rousseau, 2011 ; Livet, 2002 ; Davidson, 1991 ; Frankfurt, 2006) qu'en sociologie (Weber, 1971) ou en neurosciences (Damasio, 1995, 2003). Il va de soi aussi que cette analyse, en termes de détermination et d'affirmation de soi comme autodétermination, ne résisterait guère à une approche d'ordre psychologique. La détermination de soi par des facteurs psychologiques, c'est-à-dire en l'occurrence non sociaux, est tout aussi forte. De plus, la psychologie ne vise pas à montrer le bien-fondé de la notion de liberté, notion qui n'est pas de son registre, plutôt de celui de la philosophie. Il faut voir dans notre propos l'idée d'une liberté qui serait l'émanation d'une philosophie vulgarisée à outrance et qui peut se réduire au sentiment de liberté et à l'illusion de celle-ci par la simple affirmation du soi. Cette analyse doit bien être lue en termes d'image donnée à l'autre et à soi et ce qui est sous-tendu par le terme «liberté » dans les regards croisés de soi et de l'autre importe peu. Notre présentation qui sépare schématiquement ces deux modalités délibératives, au-delà du souci de clarification, se justifie par le fait que le regard sur l'autre tend à catégoriser celui-ci (Boudon, 1990) et le choix fait est alors considéré comme ou rationnel ou affectif, de façon tranchée.

10. «C'est mon choix » est le titre d'une émission de télévision française diffusée sur France 3, cinq jours par semaine, du 22 novembre 1999 au 18 juin 2004 et présentée par Évelyne Thomas. Cette émission, valorisant l'individu dans ce qu'il a d'original, à ses yeux en tout cas, met en avant le type de justification non rationnelle que nous décrivons ici. Ce sur quoi nous souhaitons insister est l'ampleur du phénomène médiatique, d'autres émissions reposant de façon plus ou moins évidente sur le même principe, médiatisant les processus d’individuation.

\section{Le Cas Jean-Jacques Rousseau OU L'EXPRESSION DE LA COMPLEXITÉ}

De ce qui précède, on peut, schématiquement, considérer que l'expérience de la liberté et le sentiment de liberté qui l'accompagne renvoient, d'une part, à la capacité de l'individu à se saisir de ce que propose la ville (ou plus généralement son environnement spatial et le fonctionnement social qui lui correspond), à l'utiliser à son profit et, d'autre part, à l'autonomisation de la personne, l'idéal étant d'obtenir et de tenir, autant que possible, ces deux aspects relatifs à la liberté, ce à quoi les jeunes années paraissent, jusqu'à un certain point, parvenir. Il semble cependant qu'apparaît un changement vis-à-vis de cet idéal, à partir d'un certain âge, variable selon l'individu et selon la société dans laquelle celui-ci est intégré, âge auquel l'individu est, généralement, autonome, du fait de ses capacités, et indépendant dans ses relations à autrui, donc tant sur le plan du développement psychologique que sur celui des conditions matérielles et financières. Par la complexification progressive des activités de l'individu, son autonomie ne dépend plus tant de ses capacités d'ordre technique que de ses capacités d'ordre relationnel : l'augmentation de l'autonomie, de la capacité d'action ne s'acquiert plus face aux autres mais en relation avec les autres, par la complémentarité qu'ils peuvent apporter. Le sentiment, l'impression d'indépendance peuvent alors être ainsi amoindris, l'idéal évoqué précédemment s'éloignant, devenant inaccessible.

Savoir utiliser la ville comme un ensemble d'outils que sont les aménités, savoir respecter et s'affranchir des règles de fonctionnement technique les plus simples reste accessible à chacun, de même que savoir lire une ville et s'y repérer. A contrario, savoir utiliser la ville, en tant qu'entité sociale, savoir en décrypter les règles non dites, requérant de la finesse et certaines dispositions moins partagées, savoir admettre que ces règles peuvent être utiles à condition de s'y plier n'est pas aussi aisé et bénéficier des conséquences positives du respect de ces règles, en tirer profit socialement et personnellement, revient à admettre la nécessité de certaines formes de perte d'indépendance vis-à-vis d'une partie de la société et de certains de ses représentants. Les relations entre indépendance et liberté deviennent alors complexes et la construction du rapport affectif de l'individu envers la ville s'en ressent. 
Nous explorons ici, comme illustration emblématique de la difficulté à maintenir cet équilibre, le cas de Jean-Jacques Rousseau ${ }^{11}$. Représentatif d'une époque révolue qui s'oppose, en partie à la société actuelle, plus permissive à certains égards et qui valorise l'individu et l'affirmation de soi, et particulièrement illustratif même de la difficulté de s'affirmer par autoréférencement, Rousseau nous intéresse pour montrer la complexité des relations qu'entretiennent le rapport affectif à la ville et la liberté en tant qu'elle est ressentie. S'il ne donne pas à proprement parler une définition de la liberté, s'il ne délivre pas un discours spécifiquement dédié à la liberté, il en examine néanmoins les principales facettes à travers son expérience personnelle, analysée par le regard du philosophe, même si l'individu et le philosophe sont bien évidemment indissociables : il affronte la question de la liberté tant comme individu, ce qui le conduira à écrire ses Confessions, que comme philosophe, ce qui, notamment, donnera à ses Confessions une finesse d'analyse sans pareille quoique empreinte de subjectivité, voire d'hypocrisie révélatrice. Si les sociétés française, suisse, italienne, anglaise qu'il a fréquentées ont changé depuis le milieu du XVIII ${ }^{\mathrm{e}}$ siècle, nous pouvons néanmoins postuler une certaine permanence ontologique de l'individu et que l'exemple de Rousseau est d'actualité.

Rousseau est souvent présenté comme un amoureux de la campagne, des prairies, des paysages et de la montagne qu'il a longuement et fréquemment arpentés, généralement à pied. Il nous donne des descriptions sensibles mêlant la vue, l'ouïe, l'odorat et même le goût. Par exemple : «Tous les objets que je voyais me semblaient les garants de ma prochaine félicité. Dans les maisons j’imaginais les festins rustiques; dans les prés, de folâtres jeux; le long des eaux, les bains, des promenades, la pêche; sur les arbres, des fruits délicieux; sous leurs ombres, de voluptueux tête-à-tête; sur les montagnes, des cuves de lait et de crème, une oisiveté charmante, la paix, la simplicité, le plaisir d'aller sans savoir où » (Rousseau, 1963, p. 99-100). Mais il nous semble que, relativement à la campagne, Rousseau est

11. L'analyse de ce «cas » que représente Jean-Jacques Rousseau s'appuie essentiellement sur deux textes majeurs de cet auteur : Les rêveries $d u$ promeneur solitaire et ses Confessions. Pour une analyse plus fine du rapport affectif qu'il entretient envers la campagne et la ville, nous suggérons Martouzet (2013). d'abord un hédoniste et un esthète, avant d'être un amoureux.

La question majeure reste celle des rapports éminemment complexes qu'il entretient et tisse avec la ville : l'aime-t-il ? Ne l'aime-t-il pas? La déteste-t-il ? Notre analyse montre que la réponse est plus complexe et dépasse ces trois options.

Il est clair que Rousseau a su, rapidement, apprivoiser la ville comme objet technique, comme ensemble de possibilités pratiques et matérielles : il la visite, s'y promène, s'y loge, y travaille... Il sait y rester, y trouve même du plaisir, il sait en sortir, il peut s'en passer au moins dans une certaine mesure. Plus encore, il sait être critique : il fustige la misère et la malpropreté qui règnent dans les faubourgs de Paris, il glorifie la magnificence et l'image de puissance de Turin, il pense la petite ville comme lieu de la démocratie (Jaggi, 2010). Sur le plan relationnel, c'est plus nuancé : il expérimente des rencontres plus ou moins plaisantes et il sait profiter de l'anonymat de la grande ville, il apprend les règles à respecter non pas tant celles qui permettent d'être en ville que celles qui permettent de s'inclure dans la société, dans la cité : il sait qu'il faut faire allégeance, se trouver un ou plusieurs protecteurs, qu'il faut fréquenter les salons à la mode, qu'il faut se montrer, sous le regard acéré et l'esprit impitoyable du Tout-Paris, se montrer brillant sous peine d'être ridicule. Il sait tout cela mais ne sait pas le faire : il est intellectuellement brillant, mais ne parvient pas à l'être en société. Plus il le tente, plus il est moqué, raillé - du moins le croit-il -, ce qu'il ne supporte pas. Il en a peur par anticipation et cette peur le tétanise au point que, justement, il en devient ridicule.

Par ailleurs, et c'est l'une des raisons qui le fait aimer la campagne, c'est un amoureux de la liberté. Sa situation est la suivante : il a besoin de la ville en tant qu'elle est le moyen lui donnant la pleine possibilité de montrer qu'il est brillant mais, en même temps, la ville, dans sa composante sociale, lui renvoie l'image d'un incapable, de celui qui ne sait pas se saisir de ce que lui tend la ville. Il l'aime donc puisqu'elle lui promet l'affirmation de soi et la déteste puisque, au final et par sa faute à lui, elle lui refuse cela. Cela se passe comme si elle lui tendait d'une main ce que, en fait, elle lui refuse de l'autre, mais de son fait à lui, par son incapacité à apprivoiser la ville. Pour cela, il la déteste (de la même manière qu'il a une haute estime de lui-même et, en 
même temps se déteste). C'est d'ailleurs, parce qu'il l'aime qu'il la déteste.

En d'autres termes, il est amoureux de la liberté comme capacité accrue et de la ville comme possibilité d'accroître ses capacités. Il est clair, à la lecture de ses Confessions, que Jean-Jacques Rousseau veut réussir dans le monde, mais s'il sait ce que la ville lui offre - et il lui en est reconnaissant - il se sait aussi dans l'incapacité de saisir cette liberté-là car, outre ses difficultés relationnelles, il refuse, activement, de la saisir parce qu'elle suppose, en même temps et de façon contradictoire, une perte d'indépendance vis-à-vis de ceux qui « font » la ville, ceux qui font la vie de la cité, la société dans laquelle il voudrait tant briller. Au final, ne parvenant pas à supporter cette tension, à cette double contrainte (Watzlawick et al., 1975), il se brouille avec ses protecteurs, fuit une invitation personnelle que lui fait le roi de France, se fâche avec ses amis en lesquels il voit des ennemis de sa liberté.

Rousseau est nécessairement un cas particulier. Néanmoins il illustre la grande complexité de la construction du rapport affectif à la ville, tant il montre, à travers ses textes, le jeu d'éléments constitutifs du soi et, notamment, la liberté dans ses multiples dimensions. Il est vrai que Rousseau, par ses traits de caractère, notamment sa volonté quasimaladive d'indépendance, sa timidité, son manque d'humour, est le lieu même de l'exacerbation de ces tensions et contradictions. Ce n'est qu'à la fin de sa vie, lors de ses dernières promenades à Paris qu'on pourra le sentir réconcilié avec la ville, mais c'est sans doute parce qu'il a abandonné toute volonté d'y briller et même de simplement s'y inscrire socialement, comme le souligne la première phrase des Rêveries : "Me voici donc seul sur la terre, n'ayant plus de frère, de prochain, d'ami, de société que moi-même »(Rousseau, 1963, p. 35). La vie de Rousseau montre ainsi que si les premières fois sont structurantes, la vie, ses aléas, l'apprentissage que l'on en fait peuvent remettre cela en cause, modifier le résultat de ces moments de cristallisation rapide que sont ces premières émotions spatiales. Ensuite, l'identité, l'image de soi, leur sauvegarde prennent, avec l'âge, le relai, consolidant ou modifiant les structures préalables.

\section{Conclusion}

La construction du rapport affectif de l'individu envers la ville est éminemment complexe et la déconstruction analytique des processus qui y président permet d'évoquer les facteurs y entrant, d'élaborer des figures de personnes en tant qu'elles apprécient ou non - et comment - la ville, des figures de villes en tant qu'elles sont appréciées ou non des personnes. Mais ces déconstructionsreconstructions qui opèrent en fait par catégorisation tendent à masquer la complexité de ce rapport. Rousseau est l'exemple même de cette complexité et la richesse des entretiens que nous avons pu mener, richesse qui repose d'abord sur des contradictions, parfois irréductibles, tant relatives aux discours qu'aux faits et données relatés dans ces discours, illustre aussi cela.

Peu importe - ici en tout cas - que la liberté puisse être un leurre, du fait de déterminations, que celles-ci soient d'ordre sociologique ou d'ordre psychologique, peu importe les différentes conceptions philosophiques de la liberté, le sentiment, l'impression, les ressentis qui s'y rapportent sont omniprésents, même s'ils ne sont généralement qu'implicites dans l'expression de ces ressentis.

La notion de liberté, par le flou sémantique de ses multiples acceptions, par les contradictions qu'elle comporte en elle-même, par ses limites mêmes, par la multiplicité des ressentis qui s'y rapportent, multiplicité cachée sous l'étiquette simplificatrice de «sentiment de liberté », par le fait qu'elle est tout autant rapport à soi, rapport à l'autre et rapport à ce qui environne l'individu, par le fait, surtout, qu'elle entre, via les sentiments et les émotions, dans la construction du rapport affectif à la ville, cette liberté permet d'analyser le rapport affectif à la ville, d'analyser de nouveau les entretiens, les discours et les récits qu'ils contiennent tout en gardant cette complexité.

\section{Bibliographie}

Amin A., Thrift N., 2002. Cities. Reimagining the Urban, Londres, Wiley, 192 p.

Audas N., 2011. La dynamique affective envers les lieux urbains : de l'influence des temporalités individuelles et urbaines dans la construction et l'évolution du rapport affectif, Tours, université de Tours, 505 p. 
Bochet B., 2000. Le rapport affectif à la ville, essai de méthodologie en vue de rechercher les déterminants du rapport affectif à la ville, Tours, université de Tours, $100 \mathrm{p}$.

Boudon R., 1990. L'art de se persuader des idées douteuses, fragiles ou fausses, Paris, Fayard, 458 p.

Damasio A., 1995. L'Erreur de Descartes : la raison des émotions, Paris, Odile Jacob, 396 p.

Damasio A., 2003. Spinoza avait raison : joie et tristesse, le cerveau des émotions, Paris, Odile Jacob, 346 p.

Davidson D., 1991. Paradoxes de l'irrationalité, Combas, Éditions de l'éclat, $79 \mathrm{p}$.

Dupuy J.-P., 1996. La mauvaise foi et self-deception, Raison présente, $\mathrm{n}^{\circ} 117$, [http://www.arm.asso.fr/offres/doc_inline_ src/57/JP+DUPUY+Mauvaise+foi+et+self-deception.pdf, consulté le 25.10.2012].

Elster J., 1986. Le laboureur et ses enfants, 2 essais sur les limites de la rationalité, Paris, Minuit, 199 p.

Feildel B., 2010. Espaces et projets à l'épreuve des affects. Pour une reconnaissance du rapport affectif à l'espace dans les pratiques d'aménagement et d'urbanisme, Tours, université de Tours, $647 \mathrm{p}$

Frankfurt H. G., 2006. Les raisons de l'amour, Circé, Belval, $117 \mathrm{p}$.

Gibson J., 1986. The ecological approach to visual perception, Boston, Houghton Mifflin, 332 p.

Hume D., 1991. Traité de la nature humaine. Dissertation sur les passions, Paris, Flammarion, $351 \mathrm{p}$.

JAGGI Y., 2010. Jean-Jacques Rousseau. La ville non-aimée, la Cité idéalisée, dans Salomon Cavin J., Marchand B., Antiurbain, origines et impacts de l'urbaphobie, Lausanne, PPUR, p. 29-45.

Laflamme S., 1995. Communication et émotion. Essai de microsociologie relationnelle, Paris, L'Harmattan, 192 p.

Livet P., 2002. Émotions et rationalité morale, Paris, PUF, 291 p.

LyNCH K., 1998. L'image de la cité, Paris, Dunod, 221 p.

Martouzet D., 2007. Le rapport affectif à la ville : premiers résultats, dans Paquot Th., Lussault M., Younès Ch., Habiter, le propre de l'humain, Villes, territoires et philosophie, Paris, La Découverte, p. 171-191.

Martouzet D., 2008. Figures de l'affectif urbain, dans ColleCTIF, Actes du colloque " communication au colloque Interdisciplinarité et gestion environnementale : partage d'expériences autour de la psychologie environnementale », Actes sur CDRom, Nîmes, université de Nîmes.

Martouzet D., 2010. Amour/désamour de la ville : approche individualiste, Esquisse d'une méthode générale pour l'exa- men du rapport affectif à la ville et sa construction comme objet de recherche, dans Salomon Cavin J., Marchand B., 2010, Antiurbain, origines et impacts de l'urbaphobie, Lausanne, PPUR, p. 303-322.

Martouzet D., Bailleul H., Feildel B., Gaignard G., 2010. La carte : fonctionnalité transitionnelle et dépassement du récit de vie, Natures Sciences Sociétés, vol. 18, n² 2, p. 158-170.

Martouzet D., 2014. Ville aimable, PUFR, Tours (à paraître).

Martuccelli D., 2005. Les trois voies de l'individu sociologique, EspacesTemps.net, Textuel, 08.06.2005, [http://espacestemps.net/document 1414.html, consulté le 25.10.2011].

Mathieu N., 1996. Rural et urbain : unité et diversité dans les évolutions des modes d'habiter, dans Jollivet M., Eizner N. (dir.), L'Europe et ses campagnes, Paris, Presses FNSP, p. 187-216.

Mathieu N., 2007. L'évolution des modes d'habiter : un révélateur des mutations des sociétés urbaines et rurales. Introduction, dans Luginbuhl Y. (dir.), Nouvelles ruralités, nouvelles ruralités en Europe, Bruxelles/Bern/Berlin/Frankfurt am Main/New York/Oxford/Wien, Peter Lang, p. 25-45.

Mathieu N., 2006. Repenser les modes d'habiter pour retrouver l'esprit des lieux, Genius loci face à la Mondialisation, Les nouveaux cahiers franco-polonais, $\mathrm{n}^{\circ}$ 6, p. 33-46.

Morel-Brochet A., 2006. Ville et campagne à l'épreuve des modes d'habiter. Approche biographique des logiques habitantes, Paris, université Paris 1, 572 p.

Morel-Brochet A., 2007. À la recherche des spécificités du mode d'habiter périurbain dans les représentations et les sensibilités habitantes, Norois, vol. 4, n² 205, p. 23-36.

Morel-Brochet A., Оrtar N. (dir.), 2012. La fabrique des modes d'habiter. Homme, lieux et milieux de vie, Paris, L'Harmattan, 313 p.

Paquot Th., Lussault M., Younès Ch., 2007. Habiter, le propre de l'humain, Villes, territoires et philosophie, Paris, La Découverte, 379 p.

Rousseau J.-J., 1963. Les confessions, Paris, Gallimard et Librairie Générale Française, 2 tomes, 511 p. et 507 p.

Rousseau J.-J., 1972. Les rêveries du promeneur solitaire, Paris, Gallimard, $277 \mathrm{p}$.

Rousseau J.-J., 2011. Du contrat social, suivi de Discours sur l'économie politique et de Fragments politiques, Paris, Flammarion, $256 \mathrm{p}$.

Sartre J.-P., 1999. L'être et le néant, Paris, Gallimard, 691 p.

Watzlawick P., Weakland J., Fisch R., 1975. Changements, Paradoxes et psychothérapie, Paris, Seuil, 189 p.

Weber M., 1971. Économie et société, Paris, Plon, 410 p. 